\title{
Influence of crystallographic structure on polarization reversal in polycrystalline ferroelectric/ferroelastic materials
}

Cite as: J. Appl. Phys. 125, 174101 (2019); https://doi.org/10.1063/1.5081086

Submitted: 14 November 2018 . Accepted: 11 April 2019. Published Online: 01 May 2019

J. Schultheiß, H. Kungl, and J. Koruza (i)

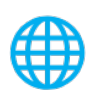

View Online

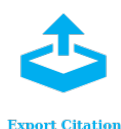

\section{ARTICLES YOU MAY BE INTERESTED IN}

Enhancement of dielectric, piezoelectric, ferroelectric, and electrocaloric properties in slightly doped ( $\left.\mathrm{Na}_{0.5} \mathrm{Bi}^{\mathrm{Bi}} .5\right)_{0.94} \mathrm{Ba}_{0.06} \mathrm{TiO}_{3}$ ceramic by samarium

Journal of Applied Physics 125, 174103 (2019); https://doi.org/10.1063/1.5083670

$\mathrm{BaTiO}_{3}$-based piezoelectrics: Fundamentals, current status, and perspectives

Applied Physics Reviews 4, 041305 (2017); https: / doi.org/10.1063/1.4990046

Fabrication and simulation investigation of zig-zag nanorod-structured graded-index antireflection coatings for LED applications

Journal of Applied Physics 125, 173102 (2019); https://doi.org/10.1063/1.5083582

\section{Journal of Applied Physics Polymer-Grafted Nanoparticles}




\title{
Influence of crystallographic structure on polarization reversal in polycrystalline ferroelectric/ferroelastic materials
}

\author{
Cite as: J. Appl. Phys. 125, 174101 (2019); doi: 10.1063/1.5081086 \\ Submitted: 14 November 2018 . Accepted: 11 April 2019 . \\ Published Online: 1 May 2019
}

\section{J. Schultheiß, ${ }^{7}$ H. Kungl, ${ }^{2}$ and J. Koruza ${ }^{7, a)}$ (D)}

\begin{abstract}
AFFILIATIONS
${ }^{7}$ Institute of Materials Science, Technische Universität Darmstadt, Alarich-Weiss-Straße 2, 64287 Darmstadt, Germany

${ }^{2}$ Institute of Energy and Climate Research, Forschungszentrum Jülich, IEK-9, 52425 Jülich, Germany
\end{abstract}

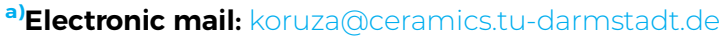

\begin{abstract}
Polarization reversal is the most fundamental physical process in ferroelectrics and directly or indirectly influences all functional properties of these materials. While this process is influenced by various intrinsic material's properties and external boundary conditions, arguably one of the most dominant parameters is the material's crystallographic structure. In this work, the influence of the crystallographic structure on the polarization reversal was investigated on the model ferroelectric system $\mathrm{Pb}(\mathrm{Zr}, \mathrm{Ti}) \mathrm{O}_{3}$ using simultaneous time-dependent polarization and strain measurements. This method enabled one to extend the understanding beyond the widely investigated relationship between the structure and coercive fields. Polarization reversal was described by three regimes, which represent a sequence of well-defined non- $180^{\circ}$ and $180^{\circ}$ switching events. The crystallographic structure was found to largely influence the mobility of the non- $180^{\circ}$ domain walls during the first switching regime, the amplitude of negative strain, and the broadness of the transition between the first and the second switching regimes, as well as the speed of the second (main) switching regime. The observed changes could be related to the amount of possible polarization directions, distribution of the local electric fields, and strain mismatch at domain wall junctions influenced by the lattice distortion. Moreover, activation fields for the first and the second regimes were experimentally determined for the investigated series of $\mathrm{Pb}(\mathrm{Zr}$, $\mathrm{Ti}) \mathrm{O}_{3}$ samples. Besides providing insight into fundamental mechanisms of polarization reversal, these results can also be used as input parameters for micromechanical or stochastic models.
\end{abstract}

\section{Published under license by AIP Publishing. https://doi.org/10.1063/1.5081086}

\section{INTRODUCTION}

Ferroelectric oxides are a vital subgroup of functional inorganic materials. Unlike nonpolar materials, ferroelectrics form a spontaneous polarization, which can be reversed by an external electric field. Unit cells with uniform polarization direction form coherent regions, which are called ferroelectric domains and are separated by domain walls. Under the application of an electric field, the polarization can be switched and domain walls can move, which gives rise to ferroelectric polarization and strain hysteresis loops. The shape of these hysteresis loops is considered as the fingerprint of a ferroelectric material. ${ }^{1}$

In most applications, ferroelectrics are utilized in their polycrystalline ceramic form. The polarization reversal process in these materials is relatively complex, since the movement of the domain walls is influenced by local electric and mechanical boundary conditions within individual grains. Other important influencing parameters are also lattice distortion, ${ }^{2}$ degree of crystallographic texture, ${ }^{3,4}$ or grain size. ${ }^{5}$ However, arguably one of the most dominating parameters is the material's crystal structure.

The influence of the crystal structure on the polarization reversal is traditionally described by comparing the coercive fields $\left(E_{c}\right)$, which are consistently reported to be lower for rhombohedral materials compared to their tetragonal counterparts. ${ }^{6-8}$ However, beyond this basic comparison, many questions remain unanswered or are giving rise to controversial reports. For example, based on theoretical calculation and experiments, the absence of the movement of non- $180^{\circ}$ domain walls was suggested for tetragonal materials, ${ }^{9}$ while others report clear experimental evidence for such processes. ${ }^{10-13}$ Moreover, little is known about the influence of the crystal structure on the mobility of non $-180^{\circ}$ domain walls under 
high electric fields or the impact on the shape of the polarization and strain loops. Under small excitation voltages, subcoercive measurements indicated a higher mobility of non- $180^{\circ}$ domain walls for rhombohedral compared to tetragonal materials, ${ }^{14,15}$ which was related to lower mechanical stresses in the former compared to the latter. ${ }^{16}$ The broader distribution of switching times for tetragonal compared to rhombohedral counterparts under switching conditions is usually related to an inhomogeneous distribution of the local electric field due to the polycrystallinity ${ }^{17}$ or to mechanical constraints due to grain-to-grain interactions. ${ }^{18}$ In addition, crystal structure dependent activation parameters accounting for individual switching events $\left(180^{\circ} /\right.$ non- $180^{\circ}$ polarization reversal) are not available in literature at all, even though these represent a fundamental input parameter for theoretical model. ${ }^{19}$

Recently, a new measurement method based on timedependent simultaneous polarization and strain measurements was suggested, which allows decrypting the polarization reversal process in more details. ${ }^{20}$ Hereafter, non- $180^{\circ}$ and $180^{\circ}$ switching events could be separated and a well-defined sequence was reported containing three regimes of the polarization reversal process. In this work, the same experimental approach is used to address the aforementioned question regarding the influence of the crystal structure on polarization switching dynamics. The study is conducted on a model ferroelectric system, polycrystalline $\mathrm{La}$-doped $\mathrm{Pb}(\mathrm{Zr}, \mathrm{Ti}) \mathrm{O}_{3}$ (PZT) with different $\mathrm{Zr} / \mathrm{Ti}$ ratios, which enables the investigation of rhombohedral (R), tetragonal (T), and morphotropic phase boundary (MPB) compositions. The new experimental method allowed us to decode the correlation between crystal structure and microstructural parameters involved in the polarization reversal process for ferroelectric/ferroelastic polycrystalline ceramic materials.

\section{MATERIALS AND METHODS}

\section{A. Materials fabrication and characterization}

$\mathrm{Pb}_{0.985} \mathrm{La}_{0.01}\left(\mathrm{Zr}_{1-x} \mathrm{Ti}_{x}\right) \mathrm{O}_{3}$ polycrystalline ceramics with different compositions $x=0.5,0.475,0.46,0.44,0.40$ across the MPB region were prepared by the mixed-oxide route, as reported elsewhere. ${ }^{21}$ Powders were pressed into cylindrical pellets with a diameter of $12 \mathrm{~mm}$ using a uniaxial pressure of $17.7 \mathrm{MPa}$, followed by cold isostatic pressing at $400 \mathrm{MPa}$. The pellets were sintered at $1050^{\circ} \mathrm{C}$ for $6 \mathrm{~h}$ with a heating rate of $2 \mathrm{~K} / \mathrm{min}$. The average grain size of the $x=0.475$ sample was determined to be $2.6 \pm 0.4 \mu \mathrm{m} .{ }^{22}$ It was previously demonstrated that the grain size is nearly independent of the $\mathrm{Zr} / \mathrm{Ti}$ ratio ${ }^{23-25}$ for the sintering conditions used in this work. We, therefore, conclude that the observed changes in the switching behavior are solely influenced by the crystallographic structure and not by the grain size. Samples were ground to a thickness of $0.7 \mathrm{~mm}$ and sputtered with platinum electrodes. X-ray diffraction (XRD) of the sintered and ground samples was performed using a X-ray diffractometer (D8 Advance, Bruker, Karlsruhe, Germany) in Bragg-Brentano geometry with an energy-dispersive $\mathrm{Si}(\mathrm{Li})$ detector and $\mathrm{CuK} \alpha$ radiation $(\lambda=0.15418 \mathrm{~nm})$ as a source.

\section{B. High voltage (HV) measurements}

The HV response was characterized independently in the frequency- and time-domain by hysteresis and pulse switching measurements, respectively. Prior to all electrical measurements, the samples were annealed at $500{ }^{\circ} \mathrm{C}$ (heating/cooling rate of $2 \mathrm{~K} / \mathrm{min}$ ) and electrically cycled 20 times at $0.5 \mathrm{~Hz}$ and $3 \mathrm{kV} / \mathrm{mm}$ to remove any unstable electrical contributions.

\section{Hysteresis measurement}

Field-dependent polarization and strain of the samples were measured by a Sawyer-Tower circuit and an optical displacement sensor (D63, Philtec Inc., Annapolis, MD, USA), respectively. A HV source (Trek Model 20/20C, Lockport, NY, USA) was used, which was remote-controlled by a LabView program. The measurement frequency was varied in the range of $0.1-2.5 \mathrm{~Hz}$.

\section{Pulse switching}

The sample was poled with $3 \mathrm{kV} / \mathrm{mm}$ for $20 \mathrm{~s}$. After poling, the sample was allowed to relax for a waiting time of $100 \mathrm{~s}$. For the switching measurement, a buffer capacitor of $2 \mu \mathrm{F}$ was combined with a commercial fast HV transistor switch (HTS 41-06-GSM, Behlke $\mathrm{GmbH}$, Kronberg, Germany), which was triggered by a function generator (Agilent 33220A, Santa Clara, CA, USA). The series of switching fields with amplitude $E_{S w}$ (varied in the range of $0.5-1.3$ times the $E_{c}$ ) was applied to the same sample. In order to check the reproducibility of the results, the measurement was repeated on two more samples. The polarization was measured by a Sawyer-Tower circuit. ${ }^{26}$ Simultaneously, the optical displacement sensor recorded the macroscopic dimensions of the sample in the direction of the applied electric field $l(t)$, which allows calculating the sample's strain $S=\left(l(t)-l_{\text {init }}\right) / l_{\text {init }}$, where $l_{\text {init }}$ is the dimension of the sample after electrical poling, before the application of the switching pulse. A detailed description of the experimental setup and the measurement sequence for dynamic polarization and strain measurements is given elsewhere. ${ }^{20}$ In order to isolate the switched polarization, $\Delta P$, from other electric contributions, a field sequence containing a switching and a reference measurement was applied. The electrical response during the "switching measurement cycle" ( $D_{S w}$, electric field antiparallel to the poling direction) contains contributions from the switched polarization, the sample's dielectric displacement, and the integrated leakage current. On the other hand, the "reference measurement cycle" $\left(D_{R e}\right.$, electric field parallel to the poling direction) only contains contributions from the latter two. The time-dependent switched polarization, $\Delta P(t)$, can be calculated by subtracting both values for any time $t: \Delta P(t)=D_{S w}(t)-D_{R e}(t){ }^{27}$

In order to ensure that the pulse switching experiment does not irreversibly impact the material, a bipolar polarization and strain loop was recorded at $3 \mathrm{kV} / \mathrm{mm}$ and $0.1 \mathrm{~Hz}$ before and after each experiment.

\section{RESULTS}

\section{A. Crystallographic and electromechanical characterization}

The (111) and (200) peaks of the XRD profiles for the investigated compositions are displayed in Fig. 1 . The $x=0.46$ sample is located at the MPB and exhibits a $\mathrm{R}$ and $\mathrm{T}$ phase coexistence. The $x=0.475$ and $x=0.5$ samples contain predominantly tetragonal $(\mathrm{T})$ microdomains, manifested by the split in the (200) peak, while the 


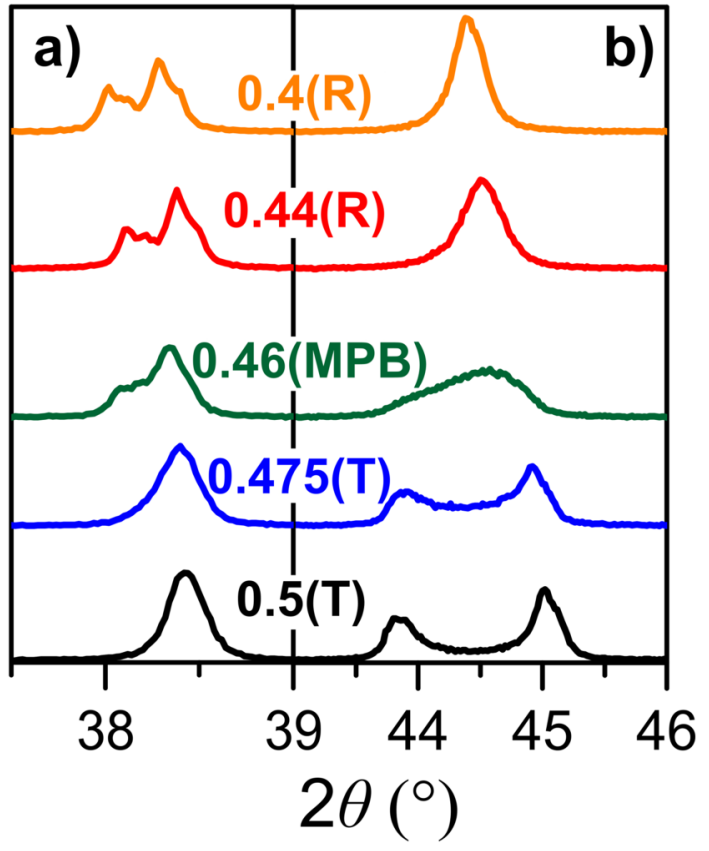

FIG. 1. (a) (111) and (b) (200) XRD peaks of polycrystalline PZT ceramics with different titanium contents $x$.

(111) peak appears as a singlet. The $x=0.44$ and $x=0.4$ samples are on the rhombohedral (R) side of the MPB. Here, the (111) peak exhibits a splitting, while the broadening of the (200) peak indicates slight distortions in the rhombohedral structure. ${ }^{28}$ The lattice distortion $^{29}$ for the tetragonal phase

$$
\delta_{t}=\frac{c}{a}-1
$$

and for the rhombohedral phase

$$
\delta_{r}=\frac{d_{111}}{d_{11 \overline{1}}}-1
$$

is displayed in Table I. While a low lattice distortion of 0.007 was found for the rhombohedral phase, the distortion of the tetragonal phase was more than two times larger. Please note that the distortion of the MPB phase could not be calculated. Similar results were previously obtained for other PZT materials. ${ }^{30}$ Results obtained by Rietveld refinement of high resolution XRD and neutron powder diffraction data on the same series of PZT materials agree to the finding. ${ }^{31}$

Large signal polarization and strain loops of PZT with different $\mathrm{Zr} / \mathrm{Ti}$ ratios are provided in Fig. 2. The characteristic properties are summarized in Table I. The highest remanent polarization was found for the $x=0.46(\mathrm{MPB})$ sample $\left(P_{r}=42.6 \mu \mathrm{C} / \mathrm{cm}^{2}\right)$. A value of $P_{r}=37.6 \mu \mathrm{C} / \mathrm{cm}^{2}$ was measured for $x=0.4(\mathrm{R})$, while a lower value of $P_{r}=32.2 \mu \mathrm{C} / \mathrm{cm}^{2}$ was found for $x=0.5(\mathrm{~T})$. The lower remanent polarization for tetragonal compositions agrees with previous theoretical calculations of switchable polarization. The maximal possible
TABLE I. Large signal ferroelectric properties (measured at $1 \mathrm{~Hz}$ and $3 \mathrm{kV} / \mathrm{mm}$ ) and the lattice distortion of polycrystalline PZT ceramics with different titanium contents.

\begin{tabular}{lccccc}
\hline \hline $\begin{array}{l}\text { Ti content } \\
x(\mathrm{~mol} \%)\end{array}$ & $\begin{array}{c}\text { Coercive } \\
\text { field } E_{c} \\
(\mathrm{kV} / \mathrm{mm})\end{array}$ & $\begin{array}{c}\text { Rem. } \\
\text { pol. } P_{r} \\
\left(\mu \mathrm{C} / \mathrm{cm}^{2}\right)\end{array}$ & $\begin{array}{c}\text { Bip. } \\
\text { strain } \\
S_{\text {bip }}(\%)\end{array}$ & $\begin{array}{c}\text { Neg. } \\
\text { strain } \\
S_{\text {neg }}^{\text {hys }}(\%)\end{array}$ & $\begin{array}{c}\text { Lattice } \\
\text { distortion } \delta\end{array}$ \\
\hline $0.5(\mathrm{~T})$ & 2.24 & 32.2 & 0.36 & -0.20 & 0.028 \\
$0.475(\mathrm{~T})$ & 1.49 & 36.5 & 0.54 & -0.34 & 0.023 \\
$0.46(\mathrm{MPB})$ & 1.12 & 42.6 & 0.54 & -0.36 & - \\
$0.44(\mathrm{R})$ & 1.10 & 40.2 & 0.44 & -0.28 & 0.007 \\
$0.4(\mathrm{R})$ & 1.09 & 37.6 & 0.34 & -0.20 & 0.007 \\
\hline \hline
\end{tabular}

fraction of switchable polarization in a polycrystalline material was calculated to be 0.831 for the $\mathrm{T}$ and 0.866 for the R phase, ${ }^{32}$ as compared to the single crystal values. In the framework of this theory, it was assumed that under high poling fields, each randomly oriented

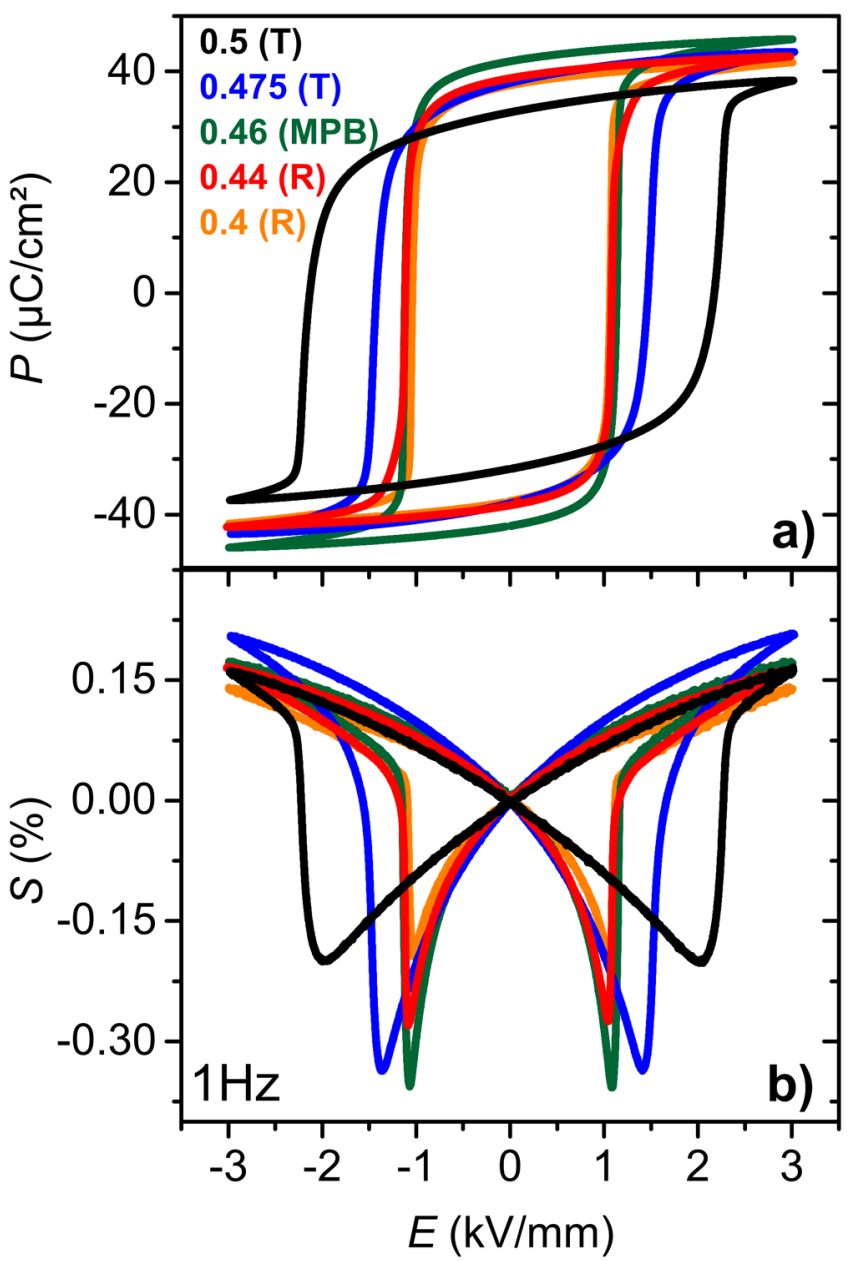

FIG. 2. Large signal (a) polarization, $P$, and (b) strain, $S$, loops as a function of the electric field, $E$, of PZT polycrystalline ceramics with different titanium contents measured at $1 \mathrm{~Hz}$. 
grain became a single domain with a polarization vector oriented in the direction of the spontaneous polarization. The values thus reflect the amount of equivalent polarization directions of 6 and 8 for the T and $\mathrm{R}$ case, respectively.

The coercive field increases with increasing the titanium content (Table I). The lowest value of $E_{c}=1.09 \mathrm{kV} / \mathrm{mm}$ was observed for the $x=0.4(\mathrm{R})$ sample, which is more than two times lower as the $E_{c}=2.24 \mathrm{kV} / \mathrm{mm}$ for the tetragonal sample $x=0.5(\mathrm{~T})$ and still $30 \%$ lower than for tetragonal PZT close to the MPB $\left(x=0.475, E_{c}=1.49 \mathrm{kV} / \mathrm{mm}\right)$. This trend is in good agreement with previously obtained results. A lower coercive field of $0.6 \mathrm{kV} / \mathrm{mm}$ was reported for a rhombohedral PZT, while a higher value of $1.2 \mathrm{kV} / \mathrm{mm}$ was found for a tetragonal composition. ${ }^{6}$ Other authors have also found higher coercive field values for the tetragonal phase compared to the rhombohedral phase in PZT. Among all investigated compositions, the negative strain and the bipolar strain peak for $x=0.46(\mathrm{MPB})$. The shape of the strain curve at the maximum negative strain is broader in the case of the tetragonal compositions, compared to the rhombohedral ones. This feature was found to be even more pronounced for undoped PZT samples. ${ }^{33}$

\section{B. Dynamic measurements}

Based on bipolar measurements (Fig. 2), it is clear that polarization switching is easier in the rhombohedral compared to the tetragonal phase. Similar observations were previously made for other materials, such as $(1-x) \mathrm{Ba}\left(\mathrm{Zr}_{0.2} \mathrm{Ti}_{0.8}\right)-x\left(\mathrm{Ba}_{0.7} \mathrm{Ca}_{0.3}\right) \mathrm{TiO}_{3}{ }^{34,35}$ or $\mathrm{BaTiO}_{3} .{ }^{36}$ Beyond this general conclusion, a more detailed understanding of the influence of the crystal structure on the polarization reversal process can be achieved if simultaneous dynamic measurements of polarization and strain are considered.
Figure 3 gives all measured data, while Fig. 4 compares three representative measurements to facilitate the discussion. The $\Delta P(t)$ curves, presented in the upper row, exhibit the expected steplike behavior. The influence of the structure can be roughly estimated from the time-dependence of the switched polarization. In order to completely switch the material in the investigated time interval of $10 \mathrm{~s}$, electric fields of $0.833 \mathrm{kV} / \mathrm{mm}$ and $0.889 \mathrm{kV} / \mathrm{mm}$ are required for $x=0.4(\mathrm{R})$ and $x=0.46(\mathrm{MPB})$ compositions, respectively, while a much larger electric field of $1.818 \mathrm{kV} / \mathrm{mm}$ needs to be applied for the $x=0.5(\mathrm{~T})$ composition.

\section{Sequence of switching mechanisms}

The simultaneous measurements of $S(t)$ are displayed in the lower row of Fig. 3. Based on the shape of the $S(t)$ curve, the sequence of switching mechanisms was divided into three regimes. ${ }^{20}$ In order to visualize the effect of the crystal structure, these regimes are highlighted in Fig. 4 by numbers for selected measurements with $\mathrm{HV}$ pulses of $E_{S w}=0.967 \mathrm{kV} / \mathrm{mm}, E_{S w}=0.994$ $\mathrm{kV} / \mathrm{mm}$, and $E_{S w}=2.006 \mathrm{kV} / \mathrm{mm}$ for the R, MPB, and T material, respectively. These electric field amplitudes correspond to $E_{S w} \approx 0.9 \cdot E_{c}$. Please note that this electric field amplitude was selected to account for the different coercive fields of the materials. The effect of crystal structure on polarization reversal in each regime will be discussed in the following.

a. Regime 1: Non- $180^{\circ}$ domain wall movement. The domain configuration in the poled state of ferroelectric/ferroelastic polycrystalline samples mainly consists of non- $180^{\circ}$ domain walls with most of the domains aligned within an angular region around the poling field direction, as found for PZT materials previously. ${ }^{37,38}$ A high fraction of non- $180^{\circ}$ domain walls is required in order to

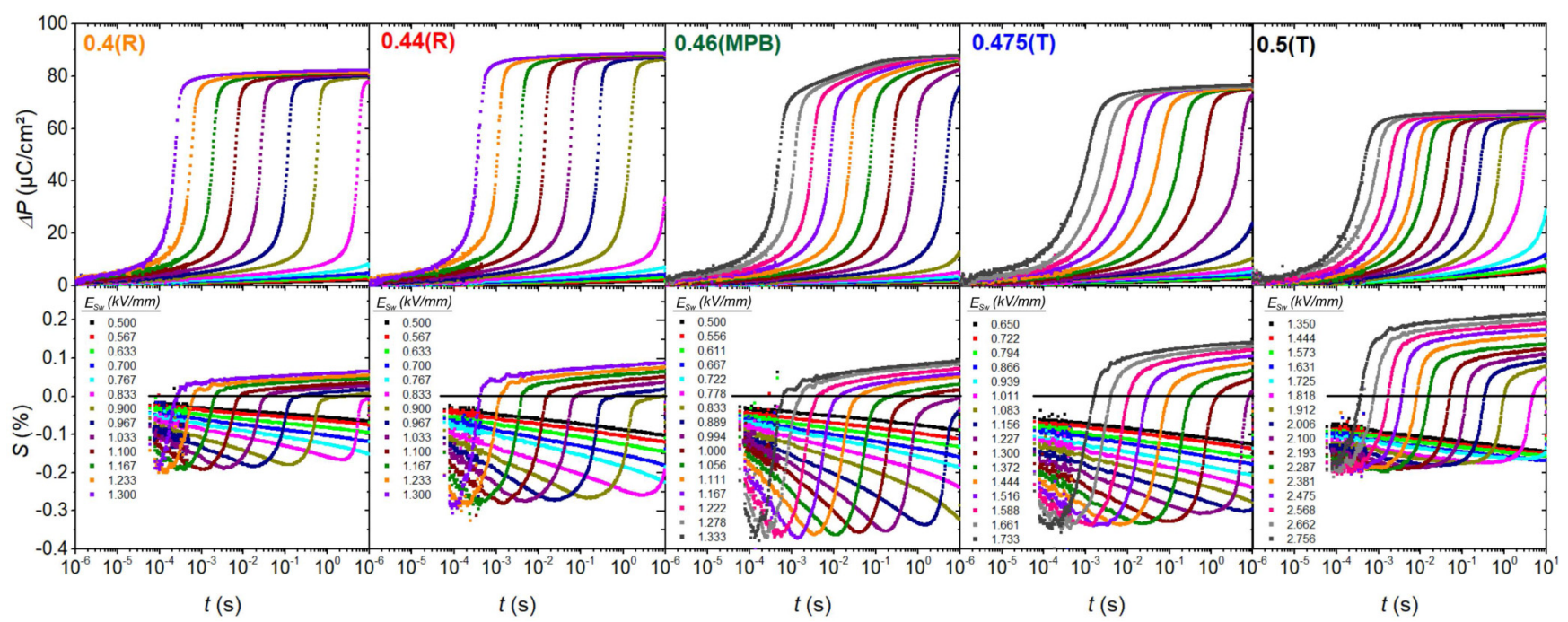

FIG. 3. Results of the simultaneous measurements of switched polarization, $\Delta P$, and strain, $S$, of polycrystalline PZT ceramics with different titanium contents. The curves were measured at different applied electric field pulses, as indicated by the inset values in $\mathrm{kV} / \mathrm{mm}$. The solid line represents zero relative strain, obtained by normalization with the poled state. All curves were measured on the same sample of the respective composition, with intermediate poling and reference measurements. 


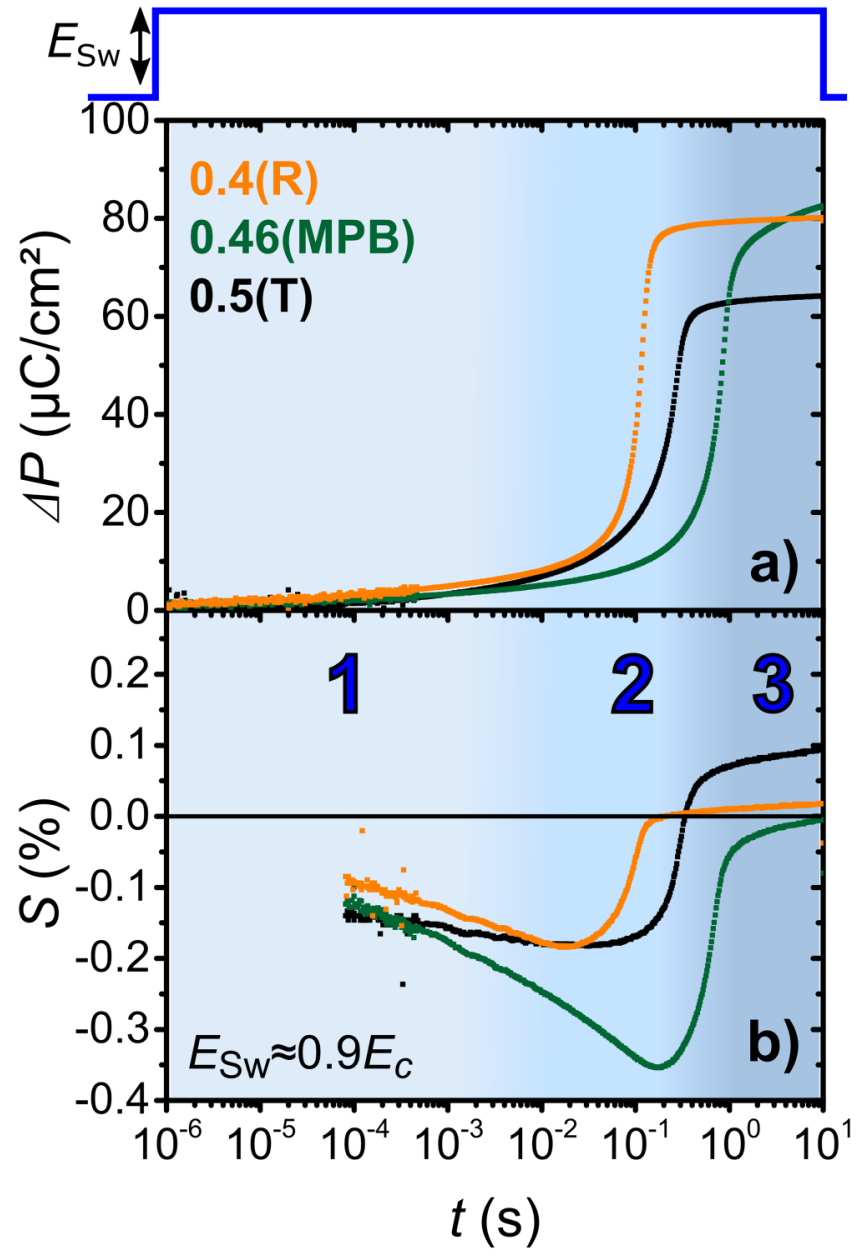

FIG. 4. Representative dynamic curves of (a) switched polarization and (b) strain to a $\mathrm{HV}$ pulse of amplitude $E_{S w}$, highlighting the influence of crystal structure on the three regimes of polarization reversal. Respective curves for a switching field of $E_{S w} \approx 0.9 \cdot E_{c}$ are displayed $\left[E_{S w}=2.006 \mathrm{kV} / \mathrm{mm}\right.$ for $x=$ $0.5(\mathrm{~T}), E_{S_{W}}=0.994 \mathrm{kV} / \mathrm{mm}$ for $x=0.46(\mathrm{MPB})$, and $E_{S_{w}}=0.967 \mathrm{kV} / \mathrm{mm}$ for $x=0.4(R)]$. The horizontal solid line in (b) represents zero strain, obtained by normalizing with the poled state. A more detailed description of the individual regimes outlined in the figure is given elsewhere.

compensate for the mechanical stresses induced by the poling process $^{39}$ and is a direct consequence of a four times lower domain wall energy of non- $180^{\circ}$ domain walls, as compared to $180^{\circ}$ domain walls. ${ }^{40}$ After applying an electric field in the opposite direction, the direct piezoelectric effect occurs and the sample shrinks instantaneously. This results in an initial negative strain at $6 \cdot 10^{-5} \mathrm{~s}$ [Fig. 4(b)]. The value is highest for the $x=0.5(\mathrm{~T})$ sample. This is related to the amplitude of the HV pulse, which is twice as high compared to the pulses for the $x=0.4(\mathrm{R})$ or $x=0.46(\mathrm{MPB})$ composition (due to different $E_{c}$ ). The subsequent linear increase of the negative strain on the logarithmic time scale, accompanied by a small increase in polarization, is related to the movement of non- $180^{\circ}$ domain walls ("non- $180^{\circ}$ domain wall movement," first regime), driven by the local electric field ${ }^{41}$ and mechanical auxiliary stresses. ${ }^{42}$ In this frame, the slope $\Delta S / \Delta \ln (t)$ reflects the influence of crystal structure on the dynamics of the non- $180^{\circ}$ domain walls. This slope-and thus the dynamics of the non- $180^{\circ}$ domain walls-is lowest for $x=0.5(\mathrm{~T})$, while it increases from $x=0.4(\mathrm{R})$ to $x=0.46(\mathrm{MPB})$. Indeed, an enhancement of the dynamics around the MPB was previously observed using small signal subcoercive AC measurements at the MPB, ${ }^{16}$ while a lower mobility was reported for tetragonal compared to rhombohedral compositions. ${ }^{14}$

The first regime ends after a field-dependent critical time is reached. This is defined as the time at which the negative strain of the sample is maximized [sign of the slope $\Delta S / \Delta \ln (t)$ changes at the transition]. The absolute amount of negative strain at this time is determined by the interplay between lattice distortion and the mobility of non $-180^{\circ}$ domain walls. These two parameters are, however, not independent and the interplay provides the maximum negative strain close to the tetragonal side of the MPB. ${ }^{43,44}$ The experimentally obtained negative strain values are $-0.18 \pm 0.01 \%$, $-0.35 \pm 0.01 \%$, and $-0.17 \pm 0.01 \%$ for $x=0.4(\mathrm{R}), x=0.46(\mathrm{MPB})$, and $x=0.5(\mathrm{~T}$ ), respectively (Fig. 3). The corresponding switched polarization values at the end of the first regime are $10.9 \pm 1.0 \mu \mathrm{C} / \mathrm{cm}^{2}$, $12.3 \pm 1.2 \mu \mathrm{C} / \mathrm{cm}^{2}$, and $11.5 \pm 0.7 \mu \mathrm{C} / \mathrm{cm}^{2}$, respectively. These values account for $13 \pm 1 \%, 14 \pm 1 \%$, and $17 \pm 1 \%$ of the totally switched polarization. The errors represent the scattering between measurements with different electric field amplitudes.

b. Regime 2: Main switching phase. After the end of the first regime, the material enters the main switching phase (regime 2, Fig. 4). The main switching phase is governed by $180^{\circ}$ switching events, accompanied by subsequent movement of non- $180^{\circ}$ domain walls. In the framework of the sequence of switching mechanisms, ${ }^{20}$ it is assumed that polarization reversal in the main switching phase requires the nucleation of a $180^{\circ}$ or a non $-180^{\circ}$ domain wall. Macroscopically, in the main switching phase, the negative strain decreases and finally turns into a positive value. This is accompanied by a large increase of the polarization. As can be observed by the $\Delta P(t)$ curves, most of the polarization is switched in the main switching phase: $63.1 \pm 1.4 \mu \mathrm{C} / \mathrm{cm}^{2}(77 \pm 2 \%), \quad 57.6 \pm 2.1 \mu \mathrm{C} / \mathrm{cm}^{2}$ $(66 \pm 2 \%)$, and $48.4 \pm 0.6 \mu \mathrm{C} / \mathrm{cm}^{2}(72 \pm 1 \%)$ for $x=0.4(\mathrm{R}), x=0.46$ (MPB), and $x=0.5(\mathrm{~T})$, respectively (Fig. 3). Compared to dynamic measurements of commercial PZT (PIC151) $)^{20}$ or other studied polycrystalline ceramic materials, ${ }^{3,23,34,35,45}$ the distribution of switching times is narrower and the rise of the $\Delta P(t)$ curve is sharper, depicting the soft doping effect of the lanthanum. ${ }^{46}$ The slope $\Delta(\Delta P) / \Delta \ln (t)$ at the inflection point of the $\Delta P(t)$ curve can be used as an approximate estimation of the switching speed. This slope is highest for $x=0.4(\mathrm{R})$ and gradually decreases with increasing titanium content (Fig. 3), indicating that switching is slower and the distribution of the switching times broader for tetragonal materials. Similarly, the $S(t)$ curves display a sharp change of strain for R and MPB materials, whereas a broader plateau can be observed for T materials (Fig. 4). This observation agrees with the bipolar strain loops, which also exhibit broadening at the transition (max. negative strain) with increasing titanium content (Fig. 2). 
c. Regime 3: Creeplike domain wall movement. The time at which the transition between regime 2 and regime 3 occurs is defined as the intersection time of the tangents of the inflection point of the $\Delta P(t)$ curve in regime 2 and the saturation of the $\Delta P(t)$ curve in regime 3 (Fig. 4). The third regime is characterized by a small increase of the macroscopic strain on the logarithmic time scale, which is accompanied by a gradual saturation of the polarization. Consequently, in this regime, only a minor amount of polarization is switched: $7.3 \pm 0.8 \mu \mathrm{C} / \mathrm{cm}^{2}(9 \pm 1 \%), 18.0 \pm 1.0 \mu \mathrm{C} / \mathrm{cm}^{2}$ $(20 \pm 1 \%)$, and $7.0 \pm 0.2 \mu \mathrm{C} / \mathrm{cm}^{2}(11 \pm 1 \%)$ for $x=0.4(\mathrm{R}), x=0.46$ (MPB), and $x=0.5(\mathrm{~T})$, respectively (Fig. 3 ).

The shape of the $\Delta P(t)$ and $S(t)$ curves in this regime resembles time-dependent measurements of polarization ${ }^{47}$ and $\operatorname{strain}^{48}$ of a poled polycrystalline ceramic material when the electric field is applied in the direction of poling. Since this behavior was previously called "electric creep" in literature, ${ }^{47,48}$ the third regime is termed "creeplike domain wall movement." The macroscopic effects in this regime were attributed to the progressive movement of the ferroelectric/ferroelastic domain walls. ${ }^{49,50}$ Please note that the physical mechanisms occurring in this regime are fundamentally different from the slow deformation of a metal or a ceramic under a mechanical load, which is also termed creep (i.e., dislocation, Nabarro-Herring, or Coble creep $^{51}$ ). For the measurements with lower electric fields in Fig. 3, the latter two regimes are absent or only partially visible within the investigated time period of $10 \mathrm{~s}$, but would appear at longer measurement times.

As displayed in Figs. 3 and 4, the curves of all $\mathrm{T}$ and $\mathrm{R}$ samples approach saturation. The higher amount of switched polarization for the MPB composition is related to the observed anomaly in $\Delta P(t)$ curves of the $x=0.46(\mathrm{MPB})$ sample, which exhibit an additional second slope in regime 3 . The origin of this behavior remains unclear. As this anomaly did not appear in other compositions, it is tempting to relate it to the presence of the MPB; however, we note that this behavior was previously observed in other polycrystalline ferroelectric samples $s^{3,34,35}$ or electrically fatigued samples. $^{52,53}$

\section{Activation fields for polarization reversal}

Field-dependent measurement of the characteristic parameters enables the determination of the activation fields of individual physical processes. The field-dependence of the characteristic parameters for the first and the second regimes is displayed in Fig. 5, whereby the error bars represent the standard deviations from three measurements on three different samples.

The field dependency of the switching times can be well described by the Merz law: ${ }^{4}$

$$
\tau=\tau_{0} \cdot \exp \left(\frac{E_{a}}{E_{S w}}\right)
$$

Here, $\tau_{0}$ is the switching time for infinite $E_{S w}$ and $E_{a}$ is the activation field for polarization reversal. The calculated activation fields are summarized in Table II and plotted as a function of the titanium content in Fig. 6 . The activation field for regime 1 (non- $180^{\circ}$ domain wall movement) is lower compared to regime 2 (main switching phase) for all compositions, except for the MPB. The low activation field of regime 1 is a consequence of the presence of

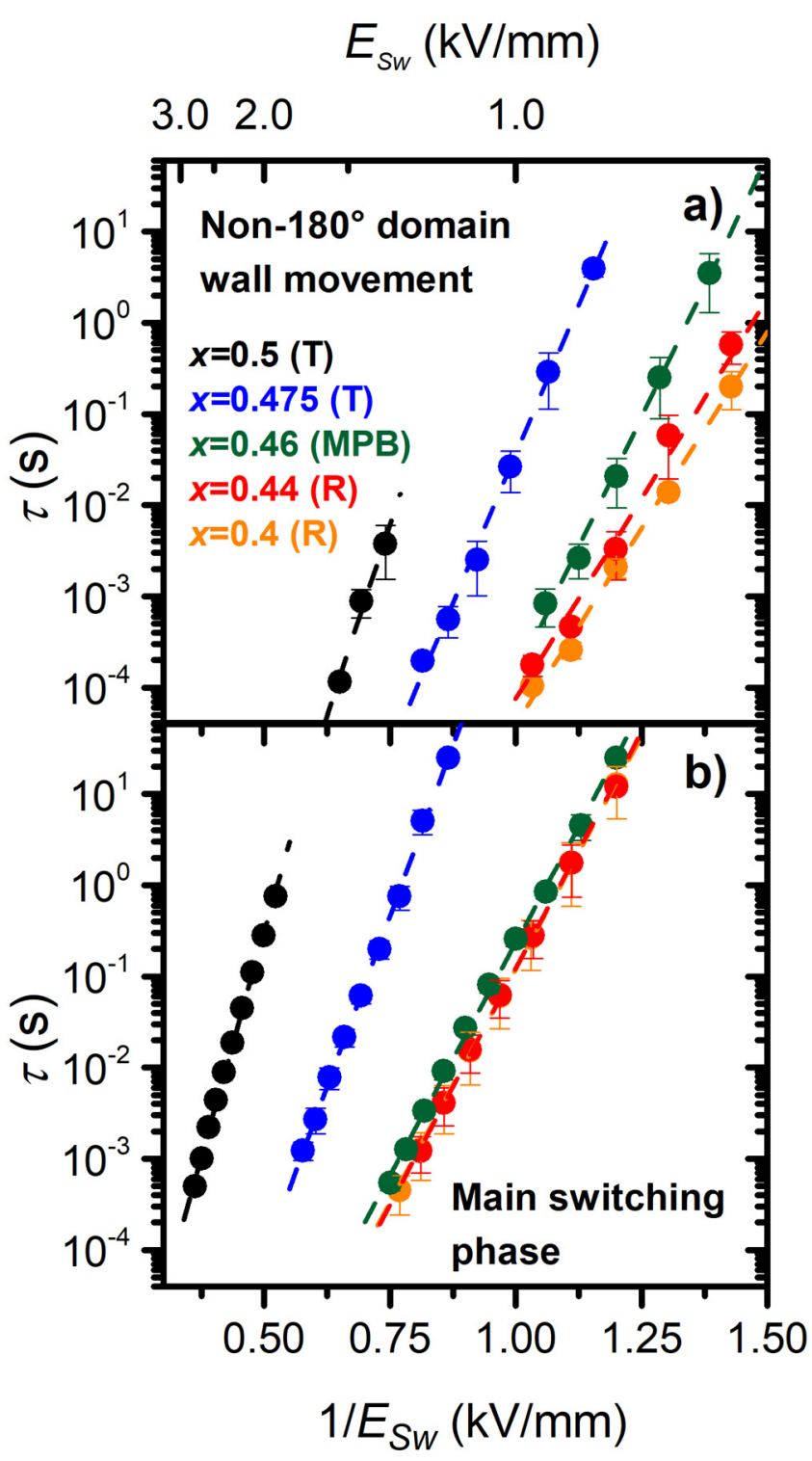

FIG. 5. Field-dependent switching times, $\tau$, for the first and second regimes: non- $180^{\circ}$ domain wall movement, and (b) main switching phase determined from the dynamic measurements in Fig. 3. The switching times were determined from (a) $S(t)$ curves (time, where $50 \%$ of the maximum negative strain is reached) and (b) $\Delta P(t)$ curves (time, where $50 \%$ of the polarization has reversed). The displayed switching times were averaged from measurements on three samples. The dashed lines represent fits according to the Merz law [Eq. (3)].

non- $180^{\circ}$ nuclei in the poled state and internal mechanical stresses, which are acting as auxiliary forces for non- $180^{\circ}$ domain wall movement in regime 1 . On the other side, in regime 2 it is assumed that nucleation is required for the $180^{\circ}$ switching event and the mechanical forces are hindering the movement of non- $180^{\circ}$ domain walls. In general, the activation fields for tetragonal 
TABLE II. Activation fields (in $\mathrm{kV} / \mathrm{mm}$ ) for non- $180^{\circ}$ domain wall movement (regime 1) and the main switching phase (regime 2).

\begin{tabular}{lcc}
\hline \hline $\begin{array}{l}\text { Ti content } \\
x(\text { mol. \%) }\end{array}$ & $\begin{array}{c}\text { Non- } 180^{\circ} \text { domain wall } \\
\text { movement (regime 1) }\end{array}$ & $\begin{array}{c}\text { Main switching } \\
\text { phase (regime 2) }\end{array}$ \\
\hline $0.5(\mathrm{~T})$ & $38.4 \pm 5.3$ & $45.4 \pm 0.7$ \\
$0.475(\mathrm{~T})$ & $30.0 \pm 1.0$ & $34.4 \pm 0.4$ \\
$0.46(\mathrm{MPB})$ & $26.3 \pm 1.0$ & $23.5 \pm 0.3$ \\
$0.44(\mathrm{R})$ & $20.2 \pm 0.9$ & $23.6 \pm 0.3$ \\
$0.4(\mathrm{R})$ & $19.8 \pm 0.5$ & $23.9 \pm 0.2$ \\
\hline
\end{tabular}

materials are by a factor of 1.5-2 higher compared to the rhombohedral counterparts.

\section{Dynamic measurements in the frequency domain}

The frequency-dependence of the bipolar polarization and strain hysteresis loops of a PZT material with $x=0.475(\mathrm{~T})$ is presented in Fig. 7(a). Similar tendencies were observed for all investigated compositions. As displayed in Fig. 7(b), the coercive field increases with increasing frequency. This feature is well established in literature ${ }^{55}$ and can also be described by state-of-the-art theoretical models (Vopsaroiu et al., ${ }^{56}$ Orihara et al., ${ }^{57}$ and $\mathrm{Du}$ and $\mathrm{Chen}^{58}$ ). Beyond the correlation between the frequency and the coercive field, also a correlation between the frequency and the negative strain exists. As displayed in Fig. 7(b), the increase of the coercive field comes along with an increase of the negative strain. These findings are in agreement with previous reports obtained from bipolar measurements. ${ }^{59-61}$ As quantified in Fig. 7(c), in agreement to the results obtained from the bipolar loops in Fig. 7(a), also the

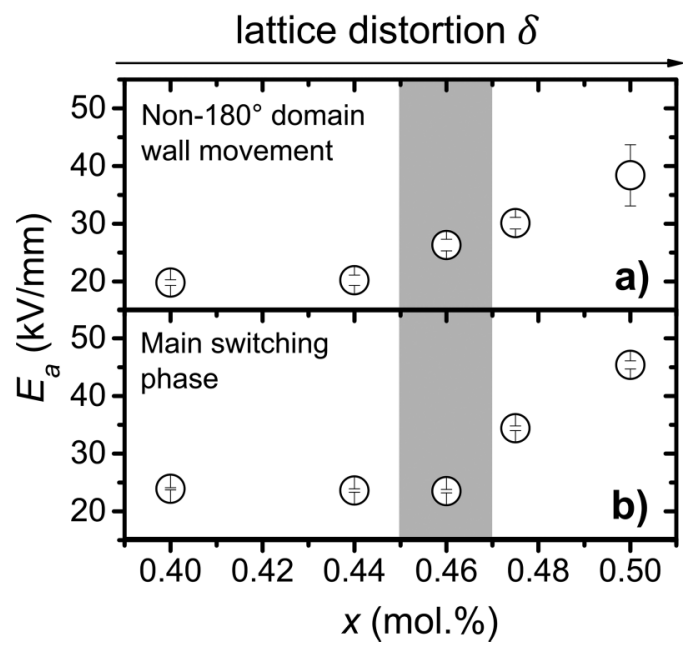

FIG. 6. Activation fields for the (a) non- $180^{\circ}$ domain wall movement (regime 1) and (b) main switching phase (regime 2) as a function of the titanium content. The error bars display the standard deviations according to the least square fit [Merz law, ${ }^{54} \mathrm{Eq}$. (3)] in Fig. 5. The arrows indicate the increasing lattice distortion (Table I). maximum negative strain obtained from the pulse measurements increases with increasing the electric field pulse amplitude for all investigated compositions (Fig. 3). This increase in strain is mainly related to the movement of non- $180^{\circ}$ domain walls. This can also be manifested by an increase in the switched polarization in regime $1\left(\Delta P_{\text {reg. } 1}\right)$, which is an indication that the share of non- $180^{\circ}$ domain switching events is time- and frequency-dependent. The share of non- $180^{\circ}$ switching events thereby increases with increasing frequency or amplitude of the applied electric field pulse, i.e., the speed of the polarization reversal process.
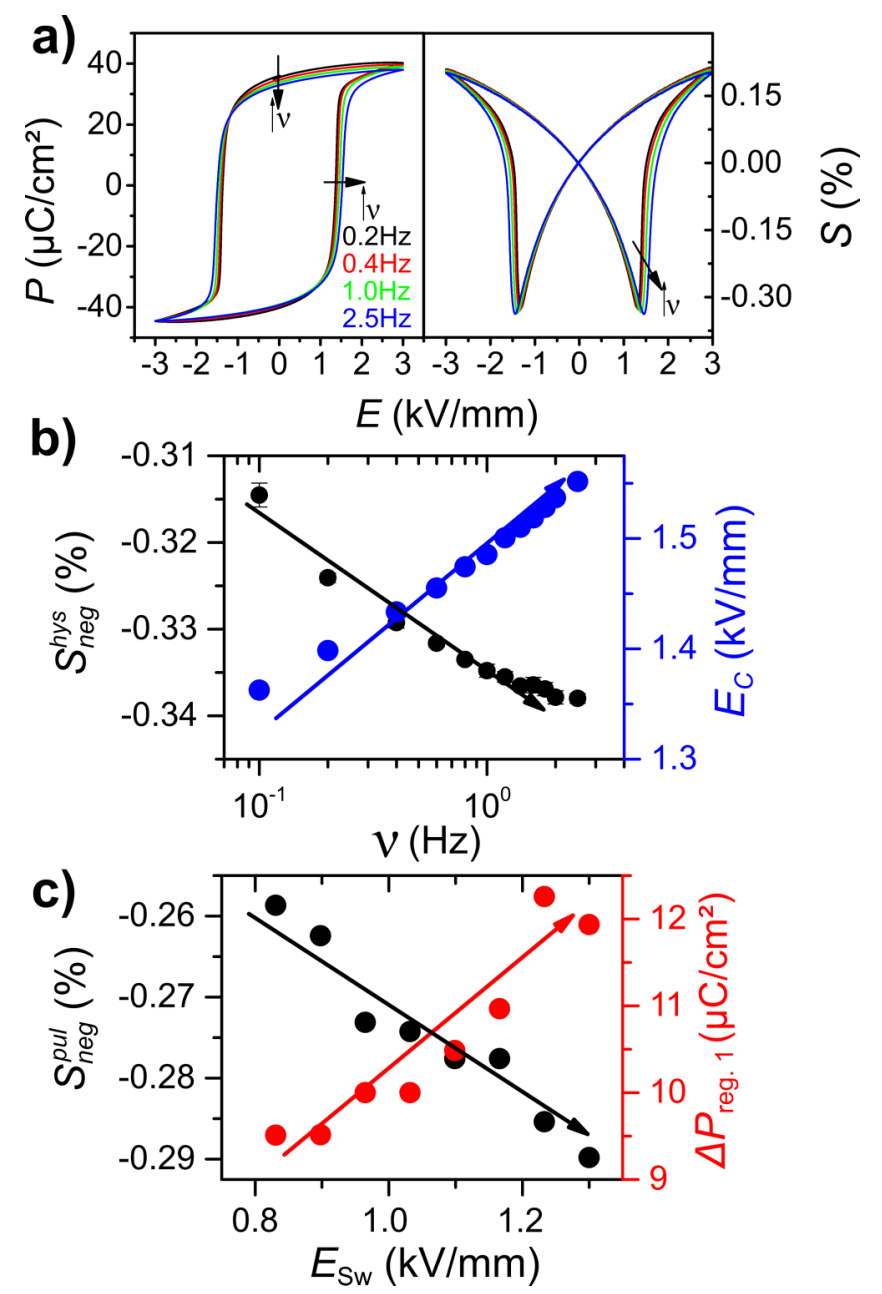

FIG. 7. Comparison between measurements in the time and frequency domain. All results are displayed for a polycrystalline PZT material with $x=0.475(T)$. (a) Frequency $(v)$-dependence of polarization and strain loops (arrows indicate the influence of frequency on the shape of the loops). (b) Frequencydependence of the negative strain obtained from hysteresis loops and the coercive field, presented in (a). (b) Correlation between the negative strain obtained from field-dependent pulse measurements (Fig. 3) and switched polarization in regime $1\left(\Delta P_{\text {reg. } 1}\right)$ and the amplitude of the electric field pulse. Arrows in (b) and $(c)$ represent a guide to the eye. Error bars are a result of values averaged from three measurements of different samples. 


\section{DISCUSSION}

Figure 8 displays different aspects which need to be considered in order to understand the obtained experimental results on the impact of crystal structure on polarization reversal. Please note that other effects, such as domain wall density or defects, are not considered in the discussion. Here, the three main aspects are discussed individually.

\section{A. Amount of possible polarization directions}

The non- $180^{\circ}$ domain walls have a lower mobility in the tetragonal compared to the rhombohedral phase (Fig. 4). As evidenced by the highest achievable negative strain (Table I) and the largest slope of the $\Delta S / \Delta t$ curve in regime 1 (Fig. 4), the highest mobility of non- $180^{\circ}$ domain walls was found for the MPB composition. The movement of a non $-180^{\circ}$ domain wall changes the shape of a grain and in this frame the easiness of non- $180^{\circ}$ domain wall movement mainly reflects the possibility of the neighboring grains to accommodate for this deformation. To some extent, this behavior resembles the plasticity of polycrystalline metallic materials, which follows Taylor's rule. The latter states that at least five independent slip systems are required for a material to be ductile and thus plastically deformable by the movement of dislocations. ${ }^{64}$ The framework of this theory was adapted to polycrystalline ferroelectric materials previously and the transformation between crystal variants or phases is treated in the same way as dislocation slip on a crystal slip system. ${ }^{65}$ To indicate the analogy, tetragonal crystals have two, while rhombohedral crystals have three independent deformation modes (ferroelectric analogy to slip systems) for the movement of non- $180^{\circ}$ domain walls, ${ }^{66}$ which is a consequence of the higher amount of equivalent polarization directions [ 6 for T, 8 for R; compare Fig. 8(a)]. The movement of non- $180^{\circ}$ domain walls would thus generate large internal stresses in tetragonal and rhombohedral materials, ${ }^{18,67}$ making such a movement unlikely. Therefore, it was previously claimed that non- $180^{\circ}$ domain wall movement is forbidden in tetragonal materials. ${ }^{9}$ However, time-resolved synchrotron diffraction experiments clearly demonstrated non- $180^{\circ}$ domain wall movement in rhombohedral ${ }^{12}$ and tetragonal ${ }^{11}$ PZT materials and the contribution of non- $180^{\circ}$ domain wall movement to the volume fraction of switched domains has been quantified to $20 \%-25 \%$ for rhombohedral and $7 \%-8 \%$ for tetragonal PZT. ${ }^{10}$ This finding is also in agreement to theoretical calculations ${ }^{68}$ and our measurements on the tetragonal materials, which give clear evidence for non- $180^{\circ}$ domain wall movement. In polycrystalline PZT compositions near the MPB, switching between the $\mathrm{R}$ and the $\mathrm{T}$ phase is possible and six independent deformation modes ${ }^{66}$ make the movement of non- $180^{\circ}$ domain walls easy.

\section{B. Lattice distortion}

As summarized in Table I, the coercive field increases with increasing titanium content. Similarly, the activation field of the first and the second regimes increases with increasing titanium content (Table II, Fig. 6). The result displayed in Fig. 6 also demonstrates that the increase of the activation fields comes along with an increasing in the lattice distortion. The correlation between lattice distortion and polarization reversal has been previously studied in literature. Easiest switching was observed for small lattice distortions, while the process got more difficult with increasing lattice distortion. ${ }^{2}$ Similar observations were also made for other PZT-based systems. ${ }^{30,69}$

On the microstructural level, a higher lattice distortion will result in a higher deviation of the angle between ferroelectric/ferroelastic domains from the theoretical value $\left(90^{\circ}\right.$ in tetragonal materials, $71^{\circ} / 109^{\circ}$ in rhombohedral materials), ${ }^{70}$ which gives rise to mechanical stresses [Fig. 8(b)]. These stresses peak at the domain junction points, where the domain structure is forced back to the theoretical angle. $^{71}$ For a lattice distortion of $\delta=0.02$, which is close to the lattice distortion of the investigated $x=0.5(\mathrm{~T})$ tetragonal sample, a maximum stress of $0.8 \mathrm{GPa}$ was found at the junction points between domains in a lamellar configuration. ${ }^{72}$ Recently, it has also been demonstrated that strained regions around domain walls may extend over several micrometers into the bulk of the polycrystalline material. ${ }^{73}$ For the movement of the domain walls, these large stresses have to be overcome. This effect is expected to be much smaller for rhombohedral materials, where only a small lattice distortion of $\delta=0.007$ was experimentally determined.
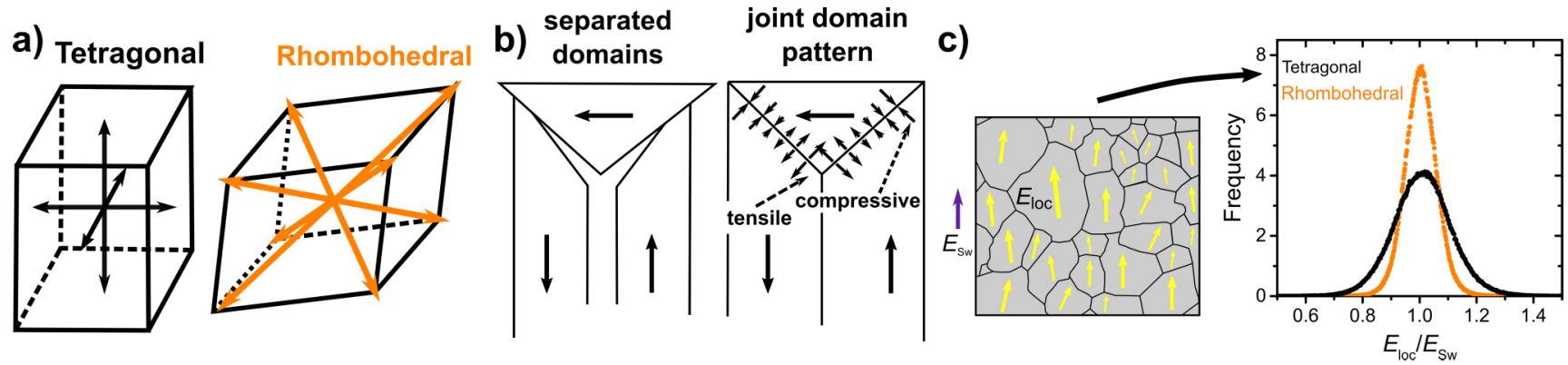

FIG. 8. Impacts of crystal structure on polarization reversal. (a) The amount of possible polarization directions is highlighted for a tetragonal and rhombohedral unit cell. (b) The separated domains show the mismatch due to the lattice distortion and the tensile and compressive strains are displayed in the joint domain pattern. (c) Calculated local electric field, $E_{\mathrm{loc}}$, distribution for a representative tetragonal and rhombohedral polycrystalline ceramic material. Values of the dielectric permittivity are taken from Ref. 62, and distribution functions of the polarization directions are taken from Ref. 63. 


\section{Distribution of the local electric field}

The externally applied electric field in the investigated materials gets locally distributed due to their polycrystalline nature ${ }^{74}$ and the broadness of the distribution of the local electric fields reflects the anisotropy in the dielectric tensor, ${ }^{75}$ which is crystal structure dependent. Based on the Landau coefficients reported by Haun et al., ${ }^{76}$ the intrinsic anisotropy $\varepsilon_{11} / \varepsilon_{33}$ is 2.5 for a tetragonal and 1.8 for a rhombohedral material. ${ }^{62}$ The obtained distributions of the local electric field are displayed in Fig. 8(c). On the one hand, tetragonal materials have a broader distribution of the local electric fields during the entire polarization reversal process $^{63}$ compared to their rhombohedral counterparts. This might explain the broadening of the switching behavior in the main switching phase as quantified by the smaller slope $\Delta(\Delta P) / \Delta \ln (t)$ at the inflection point of the $\Delta P(t)$ curve when a tetragonal material and a rhombohedral material are compared. On the other hand, the distribution of the local electric field is centered around the applied electric field and thus cannot explain the increase of the coercive field or the activation field with increasing titanium content.

\section{CONCLUSIONS}

The influence of crystal structure on the polarization reversal was studied using dynamic simultaneous measurements of polarization and strain as a response to $\mathrm{HV}$ pulses for samples with rhombohedral, tetragonal, and MPB crystal structures. These measurements allowed distinguishing between three different regimes of polarization reversal: non- $180^{\circ}$ domain wall movement, main switching phase, and creeplike movement of non- $180^{\circ}$ domain walls. The influence of the crystallographic structure on the individual regimes was described and the shape of the curves in the individual regimes allowed decrypting the governing microstructural parameters. In this context, grain-incompatibilities (non- $180^{\circ}$ domain switching induced strain-misfit between grains) and the availability of a sufficient amount of deformation modes for the movement of domain walls determine the shape of the curves in the first regime, while the inhomogeneous distribution of the local electric field and the stresses at the domains and domain junctions are more important for the shape of the curves in the main switching phase. Field-dependent measurements were also used to determine the activation fields for each event, which are important parameters for micromechanical models. ${ }^{19}$ Moreover, these measurements can be used as input for the recently developed multistep stochastic mechanism model, ${ }^{77}$ which allows quantifying the contribution of $180^{\circ}$ and non- $180^{\circ}$ switching events.

\section{ACKNOWLEDGMENTS}

This work was supported by the Deutsche Forschungsgemeinschaft (DFG) under Grant No. KO 5100/1-1. The technical expertise and valuable input of M. Weber during the construction of the HV setup is acknowledged. Y. A. Genenko and J. E. Daniels are acknowledged for valuable discussions, while R. Khachaturyan for calculating the influence of the crystal structure on the inhomogeneous field distribution.

\section{REFERENCES}

${ }^{1}$ L. Jin, F. Li, and S. J. Zhang, J. Am. Ceram. Soc. 97, 1 (2014).

${ }^{2}$ T. Leist, T. Granzow, W. Jo, and J. Rödel, J. Appl. Phys. 108, 014103 (2010).

${ }^{3}$ S. Zhukov, Y. A. Genenko, J. Koruza, J. Schultheiß, H. von Seggern, W. Sakamoto, H. Ichikawa, T. Murata, K. Hayashi, and T. Yogo, Appl. Phys. Lett. 108, 012907 (2016).

${ }^{4} J$. Schultheiß, O. Clemens, S. Zhukov, H. Seggern, W. Sakamoto, and J. Koruza, J. Am. Ceram. Soc. 100, 2098 (2017).

${ }^{5}$ C. A. Randall, N. Kim, J. P. Kucera, W. W. Cao, and T. R. Shrout, J. Am. Ceram. Soc. 81, 677 (1998).

${ }^{\mathbf{6}}$ H. Kungl and M. J. Hoffmann, J. Appl. Phys. 107, 054111 (2010).

${ }^{7}$ G. H. Haertling and W. J. Zimmer, Am. Ceram. Soc. Bull. 45, 1084 (1966).

${ }^{8}$ X. F. Chen, X. L. Dong, F. Cao, J. X. Wang, and G. S. Wang, J. Am. Ceram. Soc. 97, 213 (2014).

9. Y. Li, R. C. Rogan, E. Üstündag, and K. Bhattacharya, Nat. Mater. 4, 776 (2005).

${ }^{10}$ M. J. Hoffmann and H. Kungl, Curr. Opin. Solid State Mater. Sci. 8, 51 (2004).

${ }^{11}$ D. A. Hall, A. Steuwer, B. Cherdhirunkorn, P. J. Withers, and T. Mori, Mater. Sci. Eng. A 409, 206 (2005).

${ }^{12}$ D. A. Hall, A. Steuwer, B. Cherdhirunkorn, T. Mori, and P. J. Withers, Acta Mater. 54, 3075 (2006).

${ }^{13}$ J. L. Jones, B. J. Iverson, and K. J. Bowman, J. Am. Ceram. Soc. 90, 2297 (2007).

${ }^{14}$ J. E. García, R. Pérez, D. A. Ochoa, A. Albareda, M. H. Lente, and J. A. Eiras, J. Appl. Phys. 103, 054108 (2008).

${ }^{15}$ B. Peng, Z. X. Yue, and L. T. Li, J. Appl. Phys. 109, 054107 (2011).

${ }^{16}$ D. Damjanovic and M. Demartin, J. Phys. Condens. Matter 9, 4943 (1997).

${ }^{17}$ S. Zhukov, H. Kungl, Y. A. Genenko, and H. von Seggern, J. Appl. Phys. 115, 014103 (2014).

${ }^{18}$ Y. W. Li, X. L. Zhou, H. C. Miao, H. R. Cai, and F. X. Li, J. Appl. Phys. 113, 214111 (2013).

${ }^{19}$ S. C. Hwang, C. S. Lynch, and R. M. McMeeking, Acta Metall. Mater. 43, 2073 (1995).

${ }^{20}$ J. Schultheiß, L. Liu, H. Kungl, M. Weber, L. Kodumudi Venkataraman, S. Checchia, D. Damjanovic, J. E. Daniels, and J. Koruza, Acta Mater. 157, 355 (2018).

${ }^{\mathbf{2 1}}$ M. Hammer and M. J. Hoffmann, J. Am. Ceram. Soc. 81, 3277 (1998).

${ }^{22}$ N. Balke, H. Kungl, T. Granzow, D. C. Lupascu, M. J. Hoffmann, and J. Rödel, J. Am. Ceram. Soc. 90, 3869 (2007).

${ }^{23}$ S. Zhukov, H. Kungl, Y. A. Genenko, and H. von Seggern, J. Appl. Phys. 115, 014103 (2014).

${ }^{24}$ M. Hoffmann, M. Hammer, A. Endriss, and D. Lupascu, Acta Mater. 49, 1301 (2001).

${ }^{25}$ G. Picht, Ph.D. thesis, Karlsruhe Institute of Technology, 2013.

${ }^{26}$ C. B. Sawyer and C. Tower, Phys. Rev. 35, 269 (1930).

${ }^{27}$ H. von Seggern and S. Fedosov, IEEE Trans. Dielectr. Electr. Insul. 7, 543 (2000).

${ }^{28}$ H. Yokota, N. Zhang, A. E. Taylor, P. A. Thomas, and A. M. Glazer, Phys. Rev. B 80, 104109 (2009).

${ }^{29}$ N. Uchida and T. Ikeda, Jpn. J. Appl. Phys. 6, 1079 (1967).

${ }^{30}$ Y. H. Seo, D. J. Franzbach, J. Koruza, A. Benčan, B. Malič, M. Kosec, J. L. Jones, and K. G. Webber, Phys. Rev. B 87, 094116 (2013).

${ }^{31}$ M. Hinterstein, K. A. Schoenau, J. Kling, H. Fuess, M. Knapp, H. Kungl, and M. J. Hoffmann, J. Appl. Phys. 108, 024110 (2010).

${ }^{32}$ H. G. Baerwald, Phys. Rev. 105, 480 (1957).

${ }^{33}$ K. A. Schönau, M. Knapp, H. Kungl, M. J. Hoffmann, and H. Fuess, Phys. Rev. B 76, 144112 (2007).

${ }^{34}$ S. Zhukov, Y. A. Genenko, M. Acosta, H. Humburg, W. Jo, J. Rödel, and H. von Seggern, Appl. Phys. Lett. 103, 152904 (2013).

${ }^{35}$ S. Zhukov, M. Acosta, Y. A. Genenko, and H. von Seggern, J. Appl. Phys. 118, 134104 (2015). 
${ }^{36} \mathrm{~N}$. Wongdamnern, K. Kanchiang, A. Ngamjarurojana, S. Ananta, Y. Laosiritaworn, A. Charoenphakdee, S. Gupta, S. Priya, and R. Yimnirun, Smart Mater. Struct. 23, 085022 (2014).

${ }^{37}$ E. T. Keve and K. L. Bye, J. Appl. Phys. 46, 810 (1975).

${ }^{38}$ Z. Y. Wang, J. E. Blendell, G. S. White, and Q. Jiang, Smart Mater. Struct. 12, 217 (2003).

${ }^{39}$ D. A. Hall, A. Steuwer, B. Cherdhirunkorn, T. Mori, and P. J. Withers, J. Appl. Phys. 96, 4245 (2004).

${ }^{40}$ B. Meyer and D. Vanderbilt, Phys. Rev. B 65, 104111 (2002).

${ }^{41}$ P. Gerthsen and G. Krüger, Ferroelectrics 11, 489 (1976).

42. J. E. Daniels, C. Cozzan, S. Ukritnukun, G. Tutuncu, J. Andrieux, J. Glaum, C. Dosch, W. Jo, and J. L. Jones, J. Appl. Phys. 115, 224104 (2014).

${ }^{43}$ H. Kungl, R. Theissmann, M. Knapp, C. Baehtz, H. Fuess, S. Wagner, T. Fett, and M. J. Hoffmann, Acta Mater. 55, 1849 (2007).

${ }^{44}$ V. A. Isupov, Sov. Phys. Solid State 10, 989 (1968).

${ }^{45}$ R. Khachaturyan, S. Zhukov, J. Schultheiß, C. Galassi, C. Reimuth, J. Koruza, H. von Seggern, and Y. A. Genenko, J. Phys. D Appl. Phys. 50, 045303 (2017).

${ }^{46}$ W. L. Zhu, I. Fujii, W. Ren, and S. Trolier-McKinstry, J. Am. Ceram. Soc. 95, 2906 (2012).

${ }^{47}$ Q. D. Liu and J. E. Huber, J. Eur. Ceram. Soc. 26, 2799 (2006).

${ }^{48}$ D. Y. Zhou and M. Kamlah, J. Appl. Phys. 98, 104107 (2005).

${ }^{49}$ G. Viola, K. B. Chong, F. Guiu, and M. J. Reece, J. Appl. Phys. 115, 034106 (2014).

${ }^{50}$ T. Fett and G. Thun, J. Mater. Sci. Lett. 17, 1929 (1998).

${ }^{51}$ W. R. Cannon and T. G. Langdon, J. Mater. Sci. 18, 1 (1983).

${ }^{52}$ S. Zhukov, J. Glaum, H. Kungl, E. Sapper, R. Dittmer, Y. A. Genenko, and H. von Seggern, J. Appl. Phys. 120, 064103 (2016).

${ }^{53}$ S. Zhukov, Y. A. Genenko, O. Hirsch, J. Glaum, T. Granzow, and H. von Seggern, Phys. Rev. B 82, 014109 (2010).

${ }^{54}$ W. J. Merz, Phys. Rev. 95, 690 (1954).

${ }^{55}$ X. F. Chen, X. L. Dong, H. L. Zhang, F. Cao, G. S. Wang, Y. Gu, H. L. He, and Y. S. Liu, J. Am. Ceram. Soc. 94, 4165 (2011).
${ }^{56}$ M. Vopsaroiu, J. Blackburn, M. G. Cain, and P. M. Weaver, Phys. Rev. B 82, 024109 (2010).

${ }^{57}$ H. Orihara, S. Hashimoto, and Y. Ishibashi, J. Phys. Soc. Jpn. 63, 1031 (1994).

${ }^{58} \mathrm{X}$. Du and I. W. Chen, MRS Proc. 493, 311 (1997).

${ }^{59}$ A. Arockiarajan, A. Menzel, B. Delibas, and W. Seemann, Eur. J. Mech. A 25, 950 (2006).

${ }^{60}$ D. Zhou, M. Kamlah, and D. Munz, in Rate Dependence of Soft PZT Ceramics under Electric Field Loading (International Society for Optics and Photonics, 2001), p. 64.

${ }^{61}$ N. Lee and S. J. Kim, Ceram. Int. 38, 1115 (2012).

${ }^{62}$ X. H. Du, U. Belegundu, and K. Uchino, Jpn. J. Appl. Phys. 1 36, 5580 (1997).

${ }^{63}$ R. Khachaturyan and Y. A. Genenko, Phys. Rev. B 98, 134106 (2018).

${ }^{64}$ G. I. Taylor, J. Inst. Metals 62, 307 (1938).

${ }^{65}$ J. E. Huber, N. A. Fleck, and R. M. McMeeking, Ferroelectrics 228, 39 (1999).

${ }^{66} \mathrm{~F}$. X. Li and A. K. Soh, Acta Mater. 58, 2207 (2010).

${ }^{67}$ W. Tang, D. N. Fang, and J. Y. Li, J. Mech. Phys. Solid. 57, 1683 (2009).

${ }^{68}$ F. X. Li, X. L. Zhou, and A. K. Soh, Appl. Phys. Lett. 96, 152905 (2010).

${ }^{69}$ P. Gerber, U. Böttger, and R. Waser, J. Appl. Phys. 100, 124105 (2006).

${ }^{\mathbf{7 0}^{2}}$ S. Stemmer, S. K. Streiffer, F. Ernst, and M. Rühle, Philos. Mag. A 71, 713 (1995).

${ }^{71}$ G. Arlt and P. Sasko, J. Appl. Phys. 51, 4956 (1980).

${ }^{72}$ I. MacLaren, L. A. Schmitt, H. Fuess, H. Kungl, and M. J. Hoffmann, J. Appl. Phys. 97, 094102 (2005).

${ }^{73}$ H. Simons, A. B. Haugen, A. C. Jakobsen, S. Schmidt, F. Stöhr, M. Majkut, C. Detlefs, J. E. Daniels, D. Damjanovic, and H. F. Poulsen, Nat. Mater. 17, 814 (2018).

${ }^{74}$ D. C. Lupascu, S. Fedosov, C. Verdier, J. Rödel, and H. von Seggern, J. Appl. Phys. 95, 1386 (2004).

${ }^{75}$ Y. A. Genenko, S. Zhukov, S. V. Yampolskii, J. Schütrumpf, R. Dittmer, W. Jo, H. Kungl, M. J. Hoffmann, and H. von Seggern, Adv. Funct. Mater. 22, 2058 (2012). ${ }^{76}$ M. J. Haun, E. Furman, S. J. Jang, and L. E. Cross, Ferroelectrics 99, 63 (1989).

77Y. A. Genenko, R. Khachaturyan, J. Schultheiß, A. Ossipov, J. E. Daniels, and J. Koruza, Phys. Rev. B 97, 144101 (2018). 